\title{
Klosterwesen und Propstei Ellwangen
}

\author{
Einführung*
}

\author{
Von STEFAN WeINFurTer
}

In Klöstern leben Mönche, die sich aus der Welt zurückziehen, sich geradezu von ihr verabschieden. Diese Auskunft wird man heute gewöhnlich erhalten. Im Mittelalter war das anders ${ }^{1}$. Klöster waren bereits in fränkischer Zeit und dann insbesondere im hohen Mittelalter Kraftzentren und Erprobungsorte von Innovationen und „Labore“ neuer Lebensentwürfe, die in einer engen Wechselwirkung mit den gesellschaftlichen und politischen Ordnungskonfigurationen ihrer Zeit standen ${ }^{2}$. Daraus erwuchsen enorme Wirkkräfte auf diversen Gebieten. Die Menschen und die Landschaften in der Umgebung wurden von Klöstern zutiefst geprägt. Den Einfluss der Klöster auf die gesamte Wissens- und Bildungskultur kann man gar nicht hoch genug einschätzen. Nicht nur technische Innovationen, Effizienzsteigerungen in der Landwirtschaft, im Forstwesen und in der Fischzucht, nicht nur verbesserter Mühlenbau und arbeitsteilige Optimierung in den handwerklichen Tätigkeiten sind hier zu nennen, sondern noch viel mehr die Grundregeln, Werte und Organisationsformen menschlichen Zusammenlebens in kleinen und großen Gemeinschaften ${ }^{3}$.

* Der Beitrag basiert auf der Einführung in die gleichnamige Arbeitsgruppe, die im Rahmen der 63. Jahrestagung der Kommission für geschichtliche Landeskunde in Baden-Württemberg am 1. Juli 2016 in Ellwangen gestaltet wurde.

1 Zur Geschichte des Mönchtums Gert Melville, Die Welt der mittelalterlichen Klöster: Geschichte und Lebensformen, München 2012; Mittelalterliche Orden und Klöster im Vergleich. Methodische Ansätze und Perspektiven, hg. von Gert Melville/Anne Müller (Vita Regularis 34), Münster 2007; Ordnungskonfigurationen im hohen Mittelalter, hg. von Bernd SchneIdmüller/Stefan Weinfurter (Vorträge und Forschungen 64), Ostfildern 2006.

${ }^{2}$ Hierzu grundlegend Gert Melville, Im Spannungsfeld von religiösem Eifer und methodischem Betrieb. Zur Innovationskraft der mittelalterlichen Klöster, in: Denkströme. Journal der Sächsischen Akademie der Wissenschaften zu Leipzig, Heft 7, Leipzig 2011, S.72-92, und zusammenfassend Ders., Religiosentum - Klöster und Orden, in: Enzyklopädie des Mittelalters, hg. von Gert Melville/Martial Staub, Darmstadt 2008, S. 99-110.

3 Alfred Haverkamp, Leben in Gemeinschaft: alte und neue Formen im 12. Jahrhundert, in: Aufbruch - Wandel - Erneuerung. Beiträge zur „Renaissance“ des 12. Jahrhunderts, hg. von Georg Wieland, Stuttgart/Bad Cannstatt 1995, S.11-44, wieder gedruckt in: Alfred 
Der erste gewaltige Schub klösterlicher Wirkkraft entfaltete sich im 8. Jahrhundert. Seine Vorläufer reichen in die Spätantike Italiens ${ }^{4}$. Dort waren reich ausgestattete Bibliotheken entstanden, so im Kloster Castellum Lucullanum (die einstige Villa des Lukull) auf der Insel Megaride vor Neapel. Im 6. und 7. Jahrhundert kann man einen Aufschwung der Buchproduktion in den Klöstern im südlichen Frankreich entlang der Rhône-Achse erkennen. Besondere Bedeutung erlangte das 613/614 entstandene Kloster Bobbio in Norditalien (Provinz Piacenza), eine Gründung des irischen Missionars Columban. Dieses Kloster muss man zu den bedeutendsten Überlieferungszentren des Abendlands zählen. Eine andere Gründung Columbans war Luxueil in Burgund, ebenfalls ausgestattet mit einer reichen Bibliothek. Von dort aus wurde 662 das Königskloster Corbie gegründet. Zu Ende des 7. Jahrhunderts entstand das Königskloster Chelles an der Marne, auch dieses herausragend als Bildungszentrum.

Um die Mitte des 8. Jahrhunderts setzte eine regelrechte Flut an Klostergründungen ein ${ }^{5}$. Dies hing damit zusammen, dass sich die Normen und Werte der christlichen Lebensordnung durch verschiedene Missionsunternehmungen und durch die Förderung der politischen Elite rasch verbreiteten und in der Lebenswirklichkeit der Menschen verankerten. Könige, Hausmeier, Herzöge und Grafen nutzten die Kraft, die von den Klöstern ausging. An vorderster Stelle standen die Herzöge von Bayern, Odilo und Tassilo III., und die fränkischen Herrscher Pippin und Karl der Große. Klöster waren jetzt nicht nur Modelle christlicher Lebensführung, sondern wurden auch Marksteine der politischen Repräsentanz.

In diese Zeit fallen die Gründungen von Ellwangen, Lorsch, Fulda, Reichenau, St. Gallen, Ottobeuren, Hersfeld, Fritzlar, Ansbach, Eichstätt, Charroux, Aniane, Prüm und viele andere mehr'6. Häufig stammte die Ausstattung von Adelsfamilien. Ellwangen wurde nach der Klosterüberlieferung im Jahr 764 von Erlolf, dem Bischof von Langres, und seinem Bruder Hariolf als adliges Eigenkloster auf eigenem Grund und Boden gegründet ${ }^{7}$. Ähnlich wie Eichstätt wurde auch Ellwangen durch

Haverkamp, Gemeinden, Gemeinschaften und Kommunikationsformen im hohen und späten Mittelalter. Festgabe zur Vollendung des 65. Lebensjahres, hg. von Friedhelm BuRGard/Lukas Clemens/Michael Matheus, Trier 2002, S.207-236; Alfred Haverkamp, Bruderschaften und Gemeinden im 12. und 13. Jahrhundert, in: Ordnungskonfigurationen (wie Anm.1), S.153-192.

${ }^{4}$ Hierzu künftig Tino Licht, Halbunziale. Paläographische und philologische Studien zur ältesten lateinischen Minuskel, Habilitationsschrift masch., Heidelberg 2013.

${ }^{5}$ Karolingische Klöster. Wissenstransfer und kulturelle Innovation, hg. von Julia Becker/Tino Licht/Stefan Weinfurter (Materiale Textkulturen 4), Berlin 2015.

${ }^{6}$ Matthias Becher, Ut monasteria ... secundum ordinem regulariter vivant. Norm und Wirklichkeit in den Beziehungen zwischen Herrschern und Klöstern in der Karolingerzeit, in: ebd., S. 195-209.

7 Zu Ellwangen nach wie vor grundlegend: Ellwangen 764-1964. Beiträge und Untersuchungen zur Zwölfhundert-Jahrfeier, 2 Bände, hg. von Viktor BurR, Ellwangen 1964; 
seine Lage im alemannisch-fränkisch-bayerischen Grenzgebiet zu einem wichtigen Stützpunkt für die Karolinger in den Auseinandersetzungen mit Tassilo III., dem Herzog von Bayern. Wie andere Klöster erlangte auch Ellwangen den Schutz der Herrscher, Pippins und Karls des Großen, und wurde zu einem Königskloster. Die Regel des hl. Benedikt rückte zur Leitordnung auf, und unter Ludwig dem Frommen gab es 816/817 den Versuch, das Mönchtum im gesamten Reich auf dieser Grundlage zu vereinheitlichen. Die Klosterbibliotheken wurden zu Wissensspeichern der abendländisch-europäischen Kultur. Die überragende Bedeutung des Klosters auf der Reichenau und seine Ausstrahlung wurde erst vor kurzem in seinen sensationellen Dimensionen erkannt ${ }^{8}$. Auf Grund der engen Beziehungen, die Ellwangen mit dem Reichenaukloster pflegte, darf man durchaus von einem ähnlich hochstehenden kulturellen und theologisch-wissenschaftlichen Niveau ausgehen ${ }^{9}$, auch wenn die Zeugnisse zum größten Teil verloren sind.

Das System der frühmittelalterlichen Klosterkultur mit der weitgehend selbstständigen Einzelabtei hielt sich über einige Jahrhunderte. Im 10. und 11. Jahrhundert kam es zu Reformimpulsen, insbesondere unter Heinrich II. kurz nach der Jahrtausendwende ${ }^{10}$. Unter ihm wurde die Regel des hl. Benedikt endgültig durchgesetzt. Cluny ging zur selben Zeit einen speziellen Weg und errichtete einen weit ausgreifenden, dislozierten Großkonvent mit Ablegern in zahlreichen Prioraten. Aber im Prinzip blieb das Modell des frühmittelalterlichen Klosters unverändert. Der entscheidende Wandel kam im späten 11. und im 12. Jahrhundert ${ }^{11}$.

Es war ein neuartiger Drang nach Optimierung der Heilsvermittlung, der die Klosterwelt im hohen Mittelalter in Bewegung brachte und gewaltige Kräfte freisetzte. Dabei kann man das Kloster zunächst durchaus als einen Ort des Experimentierens bezeichnen. Neue Modelle der gottgefälligen Lebensentwürfe wurden in der klösterlichen Gemeinschaft erprobt und perfektioniert. Das Kloster wurde

Immo EBERL, Kloster Ellwangen im Umkreis seiner Gründer, in: Aus südwestdeutscher Geschichte. Festschrift für Hans-Martin Maurer, hg. von Wolfgang Schmierer/Günter Cordes/Rudolf Kiess/Gerhard TAdDEY, Stuttgart 1994, S.73-80.

8 Natalie MaAg, Alemannische Minuskel (744-846 n. Chr.). Frühe Schriftkultur im Bodenseeraum und Voralpenland (Quellen und Untersuchungen zur lateinischen Philologie des Mittelalters 18), Stuttgart 2014.

${ }^{9}$ Zur Verbrüderung der Klöster siehe Karl Schmid/Jürgen Wollasch, Die Gemeinschaft der Lebenden und Verstorbenen in Zeugnissen des Mittelalter, in: Frühmittelalterliche Studien 1 (1967) S.365-405, hier S. 377.

10 Hartmut Hoffmann, Mönchskönig und rex idiota. Studien zur Kirchenpolitik Heinrichs II. und Konrads II. (MGH Studien und Texte 8), München 1993.

11 Stefan Weinfurter, Innovation in Klöstern und Orden des hohen Mittelalters. Zusammenfassung, in: Innovation in Klöstern und Orden des Hohen Mittelalters. Aspekte und Pragmatik eines Begriffs, hg. von Mirko Breitenstein/Stefan Burkhardt/ Julia DüCKeR (Vita Regularis 48), Münster 2012, S. 297-306. 
der zentrale Ort für die Organisation der Heilswahrheit ${ }^{12}$. Die neuen Lebensentwürfe in der Klosterwelt sollten den Menschen dienen. Klöster und Orden begannen im hohen Mittelalter miteinander zu konkurrieren in der Frage, welche Lebensweise den größeren Heilswert und damit den höchsten Rang besitzt ${ }^{13}$.

Um die Überzeugungskraft zu steigern, bekam die Moral als Argumentationsinstrument eine immer stärkere Bedeutung ${ }^{14}$. ,Gut` und ,Böse` wurden zu zentralen Kategorien in der Argumentation ${ }^{15}$. Zwischen den Mönchen und den Regularkanonikern nahmen die Spannungen $\mathrm{zu}^{16}$. Hierbei spielte die Idee des Funktionswerts eine große Rolle ${ }^{17}$. Als die wichtigste Leistung in der christlichen Gesellschaft des hohen Mittelalters galt die Heilsvermittlung an die Menschen. Das war Aufgabe der Priester. Aus diesem Grund erachteten sich die nach der Augustinusregel lebenden Kanoniker allen anderen gegenüber als übergeordnet. Sie führten den Kampf gegen das Böse in der Welt und strebten nicht nur nach dem eigenen Seelenheil, sondern auch nach dem der anderen Menschen. Um sich in der Arena der Welt des Bösen zu bewähren, sollte der Einzelne als Kämpfer für das Gute im Reformstift ausgebildet werden ${ }^{18}$. Diesen Vorgang kann man als Optimierungsoffensive in der Seelsorge umschreiben. Die Bewegung der Prämonstratenser unter ihrem charismatischen Anführer Norbert von Xanten ist das treffendste Beispiel

12 Hierzu grundlegend Gert Melville, Im Spannungsfeld von religiösem Eifer und methodischem Betrieb (wie Anm. 2), und zusammenfassend Ders., Religiosentum - Klöster und Orden (wie Anm.2).

13 Exemplarisch dafür ist der ,Dialogus duorum monachorum' des Idung von Prüfening, verfasst 1153-1155: R. B. C. HuYgens, Le moine Idung et ses deux ouvrages: „Argumentum super quattuor questionibus“ et „Dialogus duorum monachorum“ (Biblioteca degli „Studi Medievali“ 41), Spoleto 1980; vgl. Adriaan H. Bredero, Le Dialogus duorum monachorum. Un rebondissement de la polémique entre Cisterciens et Cluniasiens, in: Studi Medievali 3 (1981) S. 501-586.

14 Monika Suchan, Macht verschafft sich Moral? Gewalt in der Politik der Reformpäpste (Beiträge zur Friedensethik 34), Stuttgart 2002.

15 Der Einsatz der Moral als Kampfinstrument beginnt bereits im Investiturstreit. Siehe Stefan Weinfurter, Canossa. Die Entzauberung der Welt, München ${ }^{3} 2007$, S. $135 \mathrm{ff}$.

16 Gert Melville, Zur Abgrenzung zwischen Vita canonica und Vita monastica. Das Übertrittsproblem in kanonistischer Behandlung von Gratian bis Hostiensis, in: Secundum Regulam Vivere. Festschrift für Norbert Backmund O.Praem., hg. von Gert Melville, Windberg 1978, S. 205-243.

17 Horst Funrmann, Das Papsttum zwischen Frömmigkeit und Politik - Urban II. (1088-1099) und die Frage der Selbstheiligung, in: Deus qui mutat tempora. Menschen und Institutionen im Wandel des Mittelalters. Festschrift für Alfons Becker zu seinem fünfundsechzigsten Geburtstag, hg. von Ernst-Dieter Hehl/Hubertus SeIbert/Franz STAab, Sigmaringen 1987, S. 157-172; Horst Fuhrmann, Papst Urban II. und der Stand der Regularkanoniker (Bayerische Akademie der Wissenschaften. Phil.-Hist. Klasse, Sitzungsberichte, Jahrgang 1984, Heft 2), München 1984.

18 Consuetudines canonicorum regularium Springirsbacenses-Rodenses, hg. von Stefan Weinfurter (Corpus Christianorum. Continuatio Mediaevalis 48), Turnhout 1978, $\mathbb{} 103$. 
dafür ${ }^{19}$. Auch im Südwesten des Reichs entfaltete die Reform der Prämonstratenser große Wirkkraft ${ }^{20}$.

Gegen den Anspruch der Reformkleriker, den Spitzenrang in der Kirche zu vertreten, setzten sich die Mönche zur Wehr. Die Hirsauer Bewegung des 11. und 12. Jahrhunderts kann dabei als ein Kompromiss-Modell der monastischen Frömmigkeit unter gleichzeitiger Bindung an die Adelswelt gelten ${ }^{21}$. Keine Kompromisse mehr wollten dagegen die Zisterzienser im 12. Jahrhundert eingehen ${ }^{22}$. Ihr Ideal bestand in einer unvergleichlich strengen Lebensweise, um damit den höheren Heilswert gegenüber allen anderen Reformgruppen zu dokumentieren. Der Südwesten des Reichs kann dabei als ein besonders herausstechendes Feld der Zisterzienserbewegung gelten ${ }^{23}$.

Der Kampf um die höhere Wertigkeit brachte Innovationen auf verschiedenen Ebenen hervor. Dazu gehörten die rechtliche Absicherung, die statutenhafte Ordnung des Lebens und die Normierung von „Gut“ und „Böse“ durch die Einführung eines Strafensystems ${ }^{24}$. Normierende Lebensordnungen im Sinne von

19 Stefan Weinfurter, Norbert von Xanten und die Entstehung des Prämonstratenserordens, in: Barbarossa und die Prämonstratenser (Schriften zur staufischen Geschichte und Kunst 10), Göppingen 1989, S.67-100; Werner Вомм, Neue Lebensmodelle in einer funktionalen Gesellschaft: Die Prämonstratenser im 12. Jahrhundert, in: Macht und Ordnungsvorstellungen im hohen Mittelalter. Werkstattberichte, hg. von Stefan WeINFURTER/Frank Martin Siefarth (Münchner Kontaktstudium Geschichte 1), Neuried 1998, S.169-191; Stefan Weinfurter, Norbert von Xanten und sein neuer Lebensentwurf - Gesellschaftsordnung und Wertewandel im frühen 12. Jahrhundert, in: Norbert von Xanten und der Orden der Prämonstratenser. Sammelband zur historischen Vortragsreihe im Norbertjahr 2009/2010 in Magdeburg, hg. von Clemens Dölken O. Praem, Magdeburg 2010, S. 151 -174; Studien zum Prämonstratenserorden, hg. von Irene Crusius/Helmut Flachenecker (Germania Sacra 25), Göttingen 2003.

20 Helmut Flachenecker, Die Circaria Sueviae im Lichte der neuen Prämonstratenserforschung, in: Die Stiftskirche in Südwestdeutschland. Aufgaben und Perspektiven der Forschung, hg. von Sönke Lorenz/Oliver Auge (Schriften zur südwestdeutschen Landeskunde 35), Leinfelden-Echterdingen 2003, S.123-141.

21 Hermann Jаковs, Die Hirsauer. Ihre Ausbreitung und Rechtsstellung im Zeitalter des Investiturstreits (Kölner Historische Abhandlungen 4), Köln/Graz 1961; Klaus ScHreInER, Benediktinisches Mönchtum in der Geschichte Südwestdeutschlands (Germania Benedictina 5), Augsburg 1975.

22 Gert Melville, Die Zisterzienser und der Umbruch des Mönchtums im 11. und 12. Jahrhundert, in: Norm und Realität. Kontinutität und Wandel der Zisterzienser im Mittelalter, hg. von Franz Josef Felten/Werner Rösener, Berlin 2009, S. 23 -43.

${ }^{23}$ Anfänge der Zisterzienser in Südwestdeutschland. Politik, Kunst und Liturgie im Umfeld des Klosters Maulbronn, hg. von Peter Rückert/Dieter Planck (Oberrheinische Studien 16), Stuttgart 1999; Peter RüCKERT, Von Salmannsweiler zu Salem: Gestaltung zisterziensischer Kulturlandschafft als heilsgeschichtliches Programm, in: Das Zisterzienserkloster Salem und seine Blüte unter Abt Ulrich II. von Seelfingen (1282-1311), hg. von Werner Rösener/Peter RÜCKeRT (Oberrheinische Studien 31), Ostfildern 2014, S. 19-37.

${ }^{24}$ Regula - Consuetudines - Statuta. Studi sulle fonti normative degli ordini religiosi nei secoli centrali del Medioevo. Atti del I e II Seminario internazionale di studio del Centro 
Statuten und Gesetzen begannen ihren Siegeszug. Als geradezu sensationell darf man die Erfindung des Generalkapitels durch die Zisterzienser bezeichnen ${ }^{25}$. Damit entstand erstmals in der Geschichte des Mittelalters eine über weite Räume ausgedehnte Organisation, die auf Einheit, Einheitlichkeit, Disziplin und Dauerhaftigkeit ausgerichtet war. Das Generalkapitel der Zisterzienser wurde von anderen Orden, darunter die Dominikaner ${ }^{26}$, übernommen und darf als erstes Modell einer hocheffizienten Großorganisation gelten. Alle diese Zusammenhänge und Prozesse machen deutlich, welche Kraft und Dynamik im 12. Jahrhundert von dieser Bewegung ausgegangen sind, wie sehr um neue und immer bessere, das heißt, gottgefälligere Lebensentwürfe gerungen wurde und wie intensiv der Drang nach Optimierung und Effizienzsteigerung die Menschen erfasste. Nur so ist auch zu erklären, dass in wenigen Jahrzehnten die westchristliche Welt mit einem dichten Netz von Klöstern und Reformstiften überzogen wurde.

Diese Prozesse machen auch deutlich, wie sehr die alten benediktinischen Klöster nun in Gefahr gerieten, von dieser „Optimierungsoffensive“ völlig überrollt zu werden. Nur zögerlich suchten manche von ihnen, sich dem neuen Reformgeist anzuschließen. Für Ellwangen vermutet man, dass unter Abt Adalbert I. 1136 derartige zaghafte Annäherungen an die Hirsau-Admonter Reform stattgefunden haben ${ }^{27}$. Einen anderen Weg ging das Kloster Tegernsee, das sich mit Dietramszell ein vom Haupthaus völlig separiertes, eigenes Reformstift hiel ${ }^{28}$. Aber in der Regel reagierten die alten Benediktinerabteien mit heftiger Abneigung gegenüber dem Eifer der Reformer.

Um 1200 kam noch die Idee des „Ordens ohne Haus“ hinzu. Das bedeutete, dass das Kloster als Institution und wirtschaftliches Großunternehmen völlig in Frage gestellt wurde. Es war die Idee der Franziskaner, die den Orden als die Summe der Brüder als Individuen definierte ${ }^{29}$. Wie konnten die altehrwürdigen Klöster aus dem Frühmittelalter angesichts dessen ihre Daseinsberechtigung aufrechterhalten?

italo-tedesco di storia comparata degli ordini religiosi, hg. von Cristina Andenna/Gert Melville, Münster 2005.

25 Monika R. Dihsmaier, Carta Caritatis. Verfassung der Zisterzienser. Rechtsgeschichtliche Analyse einer Manifestation monastischer Reformideale im 12. Jahrhundert (Schriften zur Rechtsgeschichte 149), Berlin 2010.

${ }^{26}$ Georgina Rosalie Galbraith, The constitution of the Dominican order. 1216 to 1260, Manchester 1925; Hans-Joachim Sснмidт, Legitimität und Innovation. Geschichte, Kirche und neue Orden im 13. Jahrhundert, in: Vita religiosa. Festschrift für Kaspar Elm zum 70. Geburtstag, hg. von Franz Josef Felten/Nikolaus Jaspert, Berlin 1999, S.371-391.

27 Marcel Beck, Quellenkritische Studien zur Geschichte der Abtei Ellwangen, in: Studien und Mitteilungen zur Geschichte des Benediktinerordens und seiner Zweige 52 (1934) S. $73-117$.

28 Sabine Buttinger, Das Kloster Tegernsee und sein Beziehungsgefüge im 12. Jahrhundert (Studien zur altbayerischen Kirchengeschichte 12), München 2004.

29 Gert Melville/Anne Müller, Franziskanische Raumkonzepte. Zur symbolischen Bedeutung des inneren und äußeren Hauses, in: Revue Mabillon 21 (2010) S. 105-138. 
Sie mussten Wege finden, sich in dieser Welt der veränderten Anforderungen zu behaupten. Manchen ist das nicht gelungen. Das stolze, mächtige Reichskloster Lorsch, das sogar den Status einer Fürstabtei erlangt hatte, wurde in der ersten Hälfte des 13. Jahrhunderts zwischen dem Fürsterzbistum Mainz und der Rheinischen Pfalzgrafschaft regelrecht zerrieben ${ }^{30}$. Andere, wie Tegernsee, retteten sich dadurch, dass sie sich in den Schutz der neuen mächtigen Landesherrn begaben. Sie wurden zu landsässigen Klöstern. Ellwangen konnte sich dagegen seit 1215 als Fürstabtei und durch eine geschickte Politik in enger Zusammenarbeit mit dem staufischen Herrscherhaus über Wasser halten. Abt Kuno von Ellwangen $(1188-1221)$ hat sich hierbei besondere Verdienste erworben. Doch im 14. Jahrhundert war auch hier der Niedergang nicht mehr aufzuhalten ${ }^{31}$. Am Ende war die Rettung nur dadurch möglich, dass sich die Abtei 1460 mit Erlaubnis des Augsburger Bischofs in eine Propstei verwandelte. Die Mönche wurden zu Kanonikern, damit zu Klerikern, die in die kirchliche Hierarchie eingeordnet waren. Das bedeutete kirchenrechtlichen Schutz, auch wenn das Stift unter dem Titel einer reichsunmittelbaren Fürstpropstei firmierte.

So zeigt sich am Beispiel der Geschichte der Klöster besonders nachdrücklich, mit welch ungeheurer Dynamik die gesellschaftlichen, geistigen und religiösen Entwicklungen im Mittelalter abgelaufen sind ${ }^{32}$. Die großen und starken Säulen der abendländischen Kultur, die Klöster der Karolingerzeit, zu denen auch Ellwangen gehörte, haben lange Zeit in Bildung, Wissenschaft und Wirtschaft die Führung innegehabt. Doch im Hochmittelalter kamen mit ungeheurer Wucht neue, innovative Modelle klösterlicher Organisation zum Tragen. Sie haben eine neue Welt der Klöster hervorgebracht und auch die Entwicklung von Ellwangen, eines Klosters „der ersten Stunde“, nachhaltig beeinflusst und in neue Bahnen gelenkt.

30 Stefan Weinfurter, Der Untergang des alten Lorsch in spätstaufischer Zeit. Das Kloster an der Bergstraße im Spannungsfeld zwischen Papsttum, Erzstift Mainz und Pfalzgrafschaft, in: Archiv für mittelrheinische Kirchengeschichte 55 (2003) S. 31-58.

31 Immo EberL, Kloster Ellwangen im Zeitalter Kaiser Ludwigs des Bayern (1314-1347), in: Ellwanger Jahrbuch 32/1 (1987/1988) S.21-34.

32 Stefan Weinfurter, Die Macht der Reformidee. Ihre Wirkkraft in Ritualen, Politik und Moral der spätsalischen Zeit, in: Religiöse Ordnungsvorstellungen und Frömmigkeitspraxis im Hoch- und Spätmittelalter. Kolloquium aus Anlass des 60. Geburtstags von Prof. Dr. Franz J. Felten am 14. und 15. Juli 2006, hg. von Jörg Rogge, Korb 2008, S. 13-39. 